\title{
Research into Differential Threshold of Packaging Design
}

\author{
Xingguo Xu and Zongye Lu
}

Department of Art \& Design, Guangxi Technological College of Machinery and Electricity Nanning, Guangxi Postcode: 530007

Keywords: Product packaging; Differential threshold; Design research

\begin{abstract}
There are four prerequisites to successfully sell a product. The first is that that product packaging should successfully attract customers. After customers are attracted by the product package, they can have a good impression of the product. Customers become interested in the product are a necessary process for the occurrence of desire to buy and transaction. Product packaging design cannot be separated from the threshold design. The difference threshold is the amount of stimulus change produced by the two differential senses, the least amount of which is the minimum difference between the two stimulus intensities. It is a measure, but is also a measure needed to identify two different stimuli of different intensity, which is called as psychophysics in psychology. This paper analyzes the design of packaging differential threshold.

Among the many goods, in order to increase the sales of a product, we must first design its packaging. Good product packaging design allows customers to bring products to a strong visual impact, which attracts the attention of customers, and further win the goodwill of customers, which is completed on the sales process in the first step. However, packaging design should not only pay attention to art, but also pay attention to being scientific to certain degree. Visual impact has a double-sided, too strong visual impact will make eyes easily over-aesthetic, making the product favorability but lower, and too weak visual impact will allow customers to ignore the main value of the product, so the visual impact must be appropriate in the packaging design.
\end{abstract}

\section{Differential Threshold Theory}

The ability to bear things is limited, so the visual conflict of product packaging to be controlled within a certain range. The visual threshold is the meaning of sustainable visual response to the human eye caused by the minimum amount of stimulation, although the vision of people beyond the threshold for which the visual conflict can be clearly felt, but for this visual stimulus Favorability will decline, and even cause discomfort. For example, in the eyes of an American, we will clearly perceive this is a Westerner, but if there is a Japanese, we cannot tell his nationality. Therefore, the human vision to a certain extent, there is a visual threshold. Differential threshold applied to product packaging design, is the human vision in the identification of two objects in the course of the two-stimulus intensity of the smallest amount of difference can be clearly and quickly identified, they stimulate the intensity of the minimum visual difference in the Product packaging design often plays a decisive role in the important.

\section{Problems Existing in Differential Threshold of Current Product Packaging}

Product packaging differential threshold is design and studied based on customers' psychological feelings. During consumption, product packaging design plays a very important role in psychological implication. Therefore, to study customers' inner feeling is an indispensable design factor in studying differential threshold. However, as China's current packaging design is not conducive to the emergence of a number of its normal development of the phenomenon, such as excessive emphasis on the acquisition of visual receptivity, but weak on the consumer psychology of people.

Visual Stimulation Does Not in Line with People's Mentality. Product package is mainly designed according to people's visual feelings, i.e., product packaging must be in line with humans' visual threshold. Although the main purpose of packaging is to attract customers' attention, the 
ultimate goal is to use such visual impact to win customers' good impression. Therefore, for the design of whatever product, the premise is people's psychological activities.

At present, in our product packaging design, a lot of product packaging are blind pursuit of new, odd and large features, and not very good to consider the product packaging to bring the visual impact of the customer is appropriate, although in a short time to The use of freshness, to attract the attention of customers, but the freshness of the goodwill is not lasting, and the packaging design process without the function of the product and the price of reasonable consideration, increased production costs, such as In snack products which have a "cigarette candy", the packaging is the sugar on the shape of the cigarette inside the package, and this snack is targeted at children, the packaging design does not take into account the child's awareness, And the degree of awareness of this shape, so that packaged snacks over time will give the child's heart have a certain impact, not only curious about cigarettes, and even premature desire for cigarettes on the child's heart and the body will cause Trauma, and the child's economic source is the parents, in a child's health considerations, parents will try to avoid the child's contact with such items.

Such packaging does not take into account of customers' specific psychological performance. Eye is the soul of the window; people have instinctive consciousness of imitation, so the visual impact is not just the behavioral impact, but more importantly, the psychological impact, so the visual difference in packaging design consumer psychology began.

Visual Stimulation Does Not Consider the Practicality of Products. The visual impact of the product packaging is indeed a customer choice of products, one of the conditions, the customer first attracted by the product packaging, packaging and then again to create a favorable impression to the customer, but from the goodwill to the customer decided to trade There is still one of the biggest factor is the practicality of the product. Customers in the purchase of products when the functional requirements of the product have its own standards, so the packaging design must be practical function of the product and packaging closely linked to the purpose of packaging does not deceive, so the packaging design must be practical close to Product function, otherwise the customer will have a feeling of distrust packaging.

For example, there is a snack called "puffed food", that is, potato chips and other snacks, because of the quality of this snack tight seal, so the use of this packaging can exaggerate the weight of the product, just from the packaging above This product is a lot of the number, but in fact most of which are the air, the real food is only a very small part, so customers often choose this kind of food will be confused by its packaging, until the transaction is completed, there will be a fooled, Thus reducing the customer's favorability of their food, this packaging is a typical way to exaggerate the packaging, packaging, see the weight and the actual component does not meet, there is the "astronomical moon cake", this moon cake Often packaging special high-end, and this packaging design officially seized the idea of a good face to consumers, gifts, can indeed draw a small number of consumers, but from the long-term development point of view, rely on packaging to raise the price of random phenomenon For the order of the market has a certain role in disrupting the $\mathrm{v}$, is not conducive to long-term development, consumers will not be after the purchase of such products once again deceived.

\section{Correct Design of Product Packaging Differential Threshold}

Correct design of packaging threshold should not be exaggerated or blindly pursue freshness, but should design the good packaging and structure.

Use Unified Text Design. The main text is the packaging of the eyes, the text on the box when the consumer close observation, you can intuitively accurate and accurate information to tell consumers goods, graphic information can tell consumers the basic profile of goods, but not in size, , The specific provisions on the shelf life, image information is limited by the size of the packaging, the structure is often the actual situation with the physical will be a little difference, this time the text is the only solution. Series of packaging elements in the design of the text requires product designers to design different products according to different fonts, structures, in order to meet the aesthetic needs of different consumers, in particular, to achieve aesthetic and practical readability of 
the two unification, not blindly pursue style Novel, structure surprisingly, while ignoring the text of the reading attribute. Therefore, in the series of packaging design of the text in addition to the writing of the law to master, we should understand the word design elements of the application of goods to study the different styles of style, shape, intrinsic, the tension of the United States, to study how to arrange the text arrangement More beautiful. In the text design connotation and extension of accurate grasp on the basis of a unified visual image of the serialization of the design will make it serialized products on the one hand get a better text on the other hand, the use of a unified font, glyph layout, Packaging to achieve a stronger visual impact. For example, Coca-Cola series of packaging design, all the text is uniform, people at a glance, vertical text layout as much as possible occupy a larger area, so that the entire text are displayed in the consumer's eyes with high degree of identity at distance.

Use a Unified Structure Layout. Although the series of packaging products may not have the same packaging, each visual element -graphics, text and color can be in a uniform layout, or graphics are represented in the same way so as to form a unified design style. But before designing packaging, a basic principle should be abode by: content determines the performance style, how the content should be how the layout of the form. For example, Apple products all the packaging series, packaging, packaging products are the front of the concrete graphics, the front side and rear side is the brand logo, while the other two sides of the product are the specific model. Uniform packaging structure, on the one hand simple atmosphere, when they are on the shelf will also have a sense of regular order, not because of the increase in the number of clutter caused by a very unparalleled

Use a Different Color for Distinguishing. Among factors affecting visual impact, color is also a very appealing language. Different colors in the psychological level will give people a different impact, so we often say that color alone can affect a person's emotions. In addition, the consumer society, different colors have been given a different character. Red represents health and vitality, yellow represents mild and bright, white represents purity and divinity. Therefore, different colors can form a different visual impact, we use the color must be based on different psychological characteristics of different consumers' different choice. In addition, if the color of similar products for a separate modification, and other visual elements are not changed circumstances, this visual impact will become more intense, when the serialization of different colors of packaging placed in the goods full of Shelves, the use of the color of the rational design and clever mix can often form a dramatic effect beyond imagination. Coca-Cola according to the different sugar content in the introduction of four different colors of canned drinks, including red on behalf of the original flavor cola, green on behalf of the low one-third of the sugar content of life Coke, white represents a very low sugar content Diet Coke, black is completely zero sugar on behalf of Coke. Through the color difference, not only increased the visual impact of the entire cola series, but also differentiate the sugar content of the cola, greatly reducing the difficulty of consumer selection, from every time when consumers need to buy different sugar content cola. If you do not understand the requirements of detailed data, then just by virtue of the different colors of packaging, you can get the desired product in an instant.

\section{Conclusion}

As an index of sensory size, differential threshold is inversely proportional to differential sensation, i.e., the greater the difference of sensory threshold, the small the sensitivity of the difference. In product design, in addition to considering the visual impact on customers' acceptance, practicality and aesthetics of product packaging should also be designed, following the principle of humans' visual threshold limit and using scientific method to package products.

\section{References}

[1] Zhou Feidie, Qi Yuechun. Application of Differential Threshold in Continuity Design of Animation Image [J]. Art Observation, 2015, (7):129. 
[2] Zhao Fengjie, Meng Zihou, Sheng Shengwo, etc. Measurement of Subjective Reverberation Sensitivity of Chinese Instrumental Music [J]. Acoustics, 2008, 33(6): 498-503.

[3] Yang Nian'en, Li Shichang, Huang Wenying, etc. Study on the Difference of Nodal Sensation and Its Neural Mechanisms [J]. Sports Science, 2014, 34(4): 41-48.

[4] Wu Shaolan. Research on Design Elements of Product Feature Identification Based on Differential Threshold [J]. Journal of Engineering Graphics, 2009, 30(4):146-149.

[5] Wang Panfeng, Fan Gang. Discussion on Threshold Limit in Non - normal Psychological Test [J] Journal of Wuhan Public Security Cadre Management College

[6] Liu Xuezhi. The Influence of Various Factors on the Time Continuous [J]. Journal of Psychology 